\title{
ACTIVIDAD PARASITARIA DE SPALANGIA ENDIUS WALKER Y MUSCIDIFURAX RAPTORELLUS KOGAN Y LEGNER (HYMENOPTERA: PTEROMALIDAE) SOBRE MUSCA DOMESTICA L.
}

\author{
Edgar INCISO ${ }^{1}$ José IANNACONE ${ }^{2}$ \\ ${ }^{1}$ Facultad de Ciencias Biológicas, Universidad Nacional Mayor de San Marcos. \\ E-mail: edi963@hotmail.com \\ ${ }^{2}$ Laboratorio de Invertebrados. Facultad de Ciencias Biológicas, Universidad Ricardo \\ Palma, Avenida Benavides 5440, Surco, Lima, PERÚ. E-mail: joseiannacone@gmail.com
}

\begin{abstract}
RESUMEN
El empleo de microavispas como parasitoides del estado pupal de Musca domestica L. surge como una alternativa importante de control biológico que podría resultar efectiva. En el presente trabajo, se realizó un estudio comparativo de la actividad parasitaria de Spalangia endius Walker y Muscidifurax raptorellus Kogan \& Legner sobre pupas de $M$. domestica en bioensayos de laboratorio en condiciones controladas de temperatura (según ensayo), humedad relativa $(75 \pm 3 \%)$ y oscuridad. Se observó la influencia de la temperatura en el ciclo biológico y en la longevidad de ambos parasitoides para lo cual se trabajó a 4 temperaturas diferentes $\left(15^{\circ} \mathrm{C}, 20^{\circ} \mathrm{C}, 25^{\circ} \mathrm{C}\right.$ y $\left.28^{\circ} \mathrm{C}\right)$ resultando $25^{\circ} \mathrm{C}$ la temperatura óptima para la crianza. A esta temperatura, se observó que la duración del ciclo biológico fue mayor en $S$. endius (22,6 días) que en $M$. raptorellus (14,8 días) y que está inversamente relacionada con la temperatura. La longevidad fue estadísticamente similar para ambas especies (20,70 días en $S$. endius y 18,60 días en $M$. raptorellus). Se determinó la capacidad de oviposición de las microavispas a $25^{\circ} \mathrm{C}$ con 20 pupas como población constante de hospedero, obteniéndose para S. endius 15 días de oviposición con un promedio de 175 pupas parasitadas, promedio mayor que el de M. raptorellus que parasitó 140 pupas en 16 días. Para ambas especies la mayor oviposición ocurrió al tercer día. Se determinó el estado fenológico de maduración de las pupas de $M$. domestica susceptibles de ser parasitadas, a $25^{\circ} \mathrm{C}$ en grupos de 50 pupas con edades de 1, 2, 3 y 4 días, siendo las pupas de dos días de maduración, las más parasitadas, obteniéndose $66,4 \%$ de parasitismo efectivo por $S$. endius y $60,2 \%$ por $M$. raptorellus $\mathrm{Se}$ determinó el número óptimo de pupas de $M$. domestica para ser expuestas a ambos parasitoides; de las nueve relaciones de parasitación con que se trabajó el número óptimo de pupas fue 10 (relación 1:10). En condiciones de laboratorio, $S$. endius resultó más efectivo en comparación con $M$. raptorellus por lo que puede considerarse como un eficiente controlador biológico de pupas de $M$. domestica.
\end{abstract}

Palabras clave: control biológico, hymenópteros, mosca doméstica, parasitoides.

\begin{abstract}
The employment of parasitoid microwasps of pupae state of Musca domestica L. emerge as an important alternative of biological control which could result effective. In the current research, a comparative study of the parasitical activity of Spalangia endius Walker and Muscidifurax raptorellus
\end{abstract}


Kogan \& Legner over pupae of $M$. domestica in laboratory bioassays was performed, under controlled condition of temperature (according to each assay), relative humidity $(75 \pm 3 \%)$ and darkness. The temperature $\left(15^{\circ} \mathrm{C}, 20^{\circ} \mathrm{C}, 25{ }^{\circ} \mathrm{C}\right.$ and $\left.28{ }^{\circ} \mathrm{C}\right)$ influence on biological cycle and longevity of both parasitoids was analyzed, being $25^{\circ} \mathrm{C}$, the optimal temperature of breeding. At this temperature, the biological cycle duration of $S$. endius (22.6 days) was greater in comparison to M. raptorellus (14.8 days). The biological cycle length was in inverse relation with temperature for both species. The longevity in both species was statistically the same for both species (20.7 days for S. endius and 18.6 days for $M$. raptorellus). The oviposition capacity at $25^{\circ} \mathrm{C}$ with 20 pupae as constant host population was analyzed, obtaining for $S$. endius, 15 days of oviposition with 175 parasitized pupae in total; by contrast M. raptorellus parasitized 140 pupae in 16 days. The highest oviposition for both species was the third day. The phenological state of maturation over susceptible pupae of $M$. domestica to be parasitized was determined, at $25^{\circ} \mathrm{C}$ in groups of 50 pupae of $1,2,3$ and 4 days old. The pupae of two days of maturation were the most parasitized obtaining $66.4 \%$ of effective parasitism for $S$. endius and $60.2 \%$ for M. raptorellus Moreover, the optimal number of pupae of housefly to be expose to both parasitoid species was determined; of the nine relations of parasitism performed (1:5 to 1:50), the optimal number of pupae was the relation 1:10. Finally, in laboratory conditions, the microwasp $S$. endius resulted being the parasitoid more effective in comparison to M. raptorellus and could be considerate an efficient biological control of pupae of $M$. domestica.

Key words: biological control, domestic fly, hymenoptera, parasitoids.

\section{INTRODUCCIÓN}

Los Muscidae son insectos cosmopolitas, consideradas como las más dañinas para el hombre y su entorno ecológico. Presentan características biológicas (como su ciclo de vida corto, alta capacidad reproductiva y de dispersión, así como la resistencia adquirida por el uso indiscriminado de los plaguicidas químicos) que las hacen importantes, desde el punto de vista de la salud pública y veterinaria (Vergara \& Jiménez 1995). En el grupo de los Muscidae, se han registrado varias especies, pero las más representativas son Musca domestica L., Stomoxys calcitrans (L.) y Haematobia irritans (L.), llamada esta última "mosca de los cuernos". Las dos primeras constituyen el $90 \%$ de la población de adultos en las explotaciones pecuarias (Vergara \& Jiménez 1995). Musca domestica vive en contacto cercano con el hombre (sinantropía) y debido a esta estrecha relación se considera que su abundancia y capacidad para transmitir enfermedades representan una amenaza para el bienestar de la humanidad, mayor que cualquier otra especie de artrópodo. Las moscas transportan organismos patógenos sobre su superficie, así como en el interior de la cavidad intestinal y los diseminan en los vómitos, regurgitaciones y heces (Keiding 1987, Atías 1995). De esta manera se convierten en vectores mecánicos potenciales de estos organismos patógenos (Manrique \& Delfín 1997). Las enfermedades transmitidas mecánicamente por $M$. domestica incluyen más de veinte siendo alguna de ellas: tifoidea, disentería amebiana, cólera, poliomielitis, hepatitis infecciosa, conjuntivitis, tracoma y lepra (Vergara \& Jiménez 1995). 
El hombre ha utilizado principalmente el control químico para combatirlas, lo que ocasiona problemas de resistencia, evasión de lugares de reposo, así como un desproporcionado incremento de los costos de producción, es por eso que se vienen buscando nuevas alternativas de control en algunos países como Canadá, Estados Unidos y México (Legner 1967). En América latina los países que más logros han obtenido en el control biológico de M. domestica son Colombia, Venezuela, México y Argentina (Zamora 1996). Las moscas son los insectos con mayor capacidad para manifestar resistencia. La ocurrencia de este fenómeno hace necesario realizar las aplicaciones más frecuentes de insecticidas y con mayores dosis, agravando aún más los problemas económicos y las consecuencias indeseables que se tiene con la utilización de estos productos (Díaz et al. 1996).

Frente al uso desmedido de los plaguicidas (que contaminan el ambiente, provocan intoxicaciones humanas y pecuarias y propician la selección de individuos resistentes), la utilización de microavispas con capacidad parasitoide del estado pupal de $M$. doméstica, surge como una alternativa importante que podría resultar muy útil, efectiva y ser una forma altamente económica de control. Además estas microavispas no son perjudiciales a los animales ni al hombre; es por ello que se está propiciando la crianza masiva de los parasitoides en laboratorios especializados, para luego realizar la liberación en los lugares afectados por plagas de moscas (Ortíz \& Torres 1983, Mullens et al. 1986, Patterson \& Rutz 1986, Meyer et al. 1991, Weinzierl \& Jones 1998). Spalangia endius Walker y Muscidifurax raptorellus Kogan \& Legner son microavispas pteromalidas, de hábitos nocturnos, cuya actividad se evidencia al ovipositar en el interior de las pupas de $M$. domestica, destruyéndolas antes que éstas se conviertan en adultas, reduciendo las poblaciones y convirtiéndose en un potencial agente de control biológico (Rutz \& Scoles 1989, Zamora 1996, Geden 1998). La gran efectividad que parecen tener $S$. endius y $M$. raptorellus como biocontroladores y ante los escasos estudios realizados en nuestro país, nos induce a profundizar los estudios sobre la actividad parasitaria de estas microavispas sobre pupas de $M$. domestica.

Por ello se planteó determinar los factores que aseguren el óptimo ciclo vital de estas dos microavispas, evaluar su capacidad de oviposición y determinar la efectividad de su parasitación en las pupas de M. domestica.

\section{MATERIAL Y MÉTODOS}

El presente trabajo se realizó en el Programa Nacional de Control Biológico (PNCB) - Servicio Nacional de Sanidad Agraria (SENASA), Vitarte, Lima, Perú y en el Laboratorio de Inmunología Parasitaria y Epidemiología de la Facultad de Ciencias Biológicas, Universidad Nacional Mayor de San Marcos (UNMSM). Los 
bioensayos se realizaron con temperaturas controladas: $15^{\circ} \mathrm{C}, 20^{\circ} \mathrm{C}, 25^{\circ} \mathrm{C}$ y $28^{\circ} \mathrm{C}$ (determinación de la influencia de la temperatura en el ciclo biológico y longevidad) y $25^{\circ} \mathrm{C}$ (demás bioensayos), en todos los casos se mantuvo $75 \pm 3 \% \mathrm{HR}$.

\section{Crianza y mantenimiento de los parasitoides}

Los parasitoides fueron recuperados a partir de pupas de $M$. domestica colectados en establos de la Universidad Nacional Agraria - La Molina, con la ayuda de cernidores y transportadas hasta el laboratorio. La población de moscas emergidas fue usada para la crianza masiva de hospederos. Los parasitoides recuperados fueron identificados como S. endius y M. raptorellus. La crianza de los parasitoides adultos, así como los ensayos, se hicieron en oscuridad y por separado, en jaulas con dos mangas especialmente acondicionadas. En dichas jaulas, se colocaron diariamente, pupas de 1 a 2 días (pupas frescas) de la mosca para ser parasitadas, previamente limpiadas con corriente de aire y una brocha, para eliminar partículas del salvado y retiradas para la emergencia de los nuevos parasitoides. A las dos semanas, la pupas fueron limpiadas con la ayuda de un ventilador para eliminar las moscas emergidas y sus puparios las mismas que no representan el $20 \%$ del total de pupas expuestas al parasitismo.

Los parasitoides adultos, machos y hembras, a los pocos minutos de emerger ya estaban capacitados para copular y se les dio el alimento consistente en miel de abeja sin diluir, con ayuda de un pincel $\mathrm{N}^{\circ} 1$. Para continuar con el ciclo de las avispas, se les expuso nuevamente pupas frescas de $M$. domestica con el objeto de obtener nueva generación de parasitoides.

\section{Crianza y mantenimiento de la población de $M$. domestica}

En una sala de $60 \mathrm{~m}^{2}$ se trabajó con dos jaulas de crianza de dos mangas, cuyas dimensiones fueron $105 \times 58 \times 50 \mathrm{~cm}$. En el interior se colocó diariamente el alimento consistente en miel de abeja en una dilución al $10 \%$, embebida en algodón; además de leche en polvo para mejorar su oviposición y una dieta especial con salvado de trigo fermentado. Después del período de cópula, se observó el período de oviposición, depositando sus huevecillos (blanquecinos, alargados de $1 \mathrm{~mm}$ de longitud) en masas sobre el sustrato preparado con salvado de trigo fermentado, y separado en varias placas. Las placas con el salvado de trigo fermentado fueron renovadas cada $24 \mathrm{~h}$ a partir del quinto día de emergencia hasta el último día de oviposición. Después los huevos fueron sembrados en la dieta de las larvas, para su eclosión. Las larvas se mantuvieron en un ambiente ventilado. Los factores ambientales determinantes fueron la temperatura, humedad relativa y luz. La dieta para las larvas de $M$. domestica consistió en salvado de trigo fermentado por 48 horas, en una relación de 2,5 $\mathrm{kg}$ de salvado de trigo mas $2 \mathrm{~mL}$ de $\mathrm{HCl}$ al $100 \%$ y 1,5 L de agua potable. El salvado se mezcló hasta quedar muy húmedo, posteriormente 
se dejó fermentar por $48 \mathrm{~h}$, controlando la cantidad de agua presente en la dieta. Las larvas pasaron por tres estadíos, con una duración de 5 a 7 días, después se trasladó la dieta a un bastidor con malla de plástico de $2 \mathrm{~mm}$ entre hilos con las medidas 42 x $30 \times 4 \mathrm{~cm}$. El bastidor fue colocado sobre una bandeja de plástico de $42 \times 30 \times 6$ $\mathrm{cm}$, conteniendo una delgada capa de arena limpia, se esperó un lapso de 24 a $48 \mathrm{~h}$ para que las larvas ingresen a la arena. Después de esto, se tamizó la arena obteniéndose pupas limpias.

\section{Infección experimental sobre los estadíos pupales}

La aplicación de los parasitoides a los puparios de $M$. domestica es similar para ambas especies estudiadas. Las pupas frescas (de 24 a $48 \mathrm{~h}$ ) destinadas a la parasitación, fueron colocadas en vasos de plástico de $80 \mathrm{~mL}$ de capacidad y cubiertas con organza junto con los parasitoides machos y hembras (en proporción 1:1) además del alimento consistente en miel de abeja sin diluir; el ambiente de trabajo se mantuvo en oscuridad ya que estas especies de hymenópteros tienen una mayor actividad parasitaria en estas condiciones. Las pupas de donde no emergieron moscas fueron separadas ya que habrían sido parasitadas. Después que cumplieron su ciclo biológico, las avispas emergieron de los puparios y se procedió a contabilizar la cantidad de parasitoides emergidos para cada especie.

\section{Influencia de la temperatura en la crianza de los parasitoides (ciclo biológico y longevidad)}

Se desarrolló el ciclo de vida completo de $S$. endius y $M$. raptorellus a diferentes temperaturas: $15^{\circ} \mathrm{C}, 20^{\circ} \mathrm{C}, 25^{\circ} \mathrm{C}$ y $28^{\circ} \mathrm{C}$. utilizando una población de 100 pupas de $M$. domestica para cada temperatura.

\section{Capacidad de oviposición diaria de $S$. endius y M. raptorellus en condiciones controladas de laboratorio}

Se trabajó a $25^{\circ} \mathrm{C}$, con 20 pupas como población constante del hospedero. Para la crianza se utilizaron vasos descartables de $80 \mathrm{~mL}$ de capacidad. En cada vaso se colocaron 20 pupas con una pareja de parasitoides recién emergidos, los recipientes fueron cubiertos con organza y sujetados con liga, se garantizó la alimentación con finas pinceladas de miel de abeja. Se trabajó en condiciones de oscuridad (fotoperíodo: 0 - 24 h). Los cambios de hospederos se efectuaron cada $24 \mathrm{~h}$, durante la longevidad de las hembras. Los machos muertos antes que las hembras fueron reemplazados para mantener las parejas, y se anotó el número de parasitoides emergidos.

\section{Período pupal óptimo de $M$. domestica para ser parasitada}

Se realizaron observaciones para determinar en que día la pupa de $M$. domestica preferentemente sería parasitada por el parasitoide (oviposición). En cada vaso se 
tomaron grupos de 50 pupas con edades de 1, 2, 3 y 4 días, las que fueron expuestas por 24 horas y a $25^{\circ} \mathrm{C}$ a las avispas hembras a quienes se les alimentó diariamente con miel de abeja pura. Se hizo un seguimiento a las pupas de las moscas hasta obtener los parasitoides.

\section{Número óptimo de pupas para exponer a los parasitoides}

Se trabajó con las siguientes relaciones de parasitación: 1:5, 1:10, 1:15, 1:20, $1: 25,1: 30,1: 35,1: 40$ y 1:50, colocando para cada relación una pareja de parasitoides. Los machos muertos, antes que las hembras, fueron reemplazados para mantener las parejas. Cada grupo fue observado hasta la emergencia del parasitoide y se registró el número de pupas parasitadas por hembra.

\section{Análisis estadístico}

Los datos obtenidos de la duración del ciclo biológico y longevidad fueron transformados al logaritmo $\mathrm{x}+1$ para ajustarlos a la distribución normal. Establecida la significancia estadística del ANDEVA; se realizó la prueba de Tukey $(\mathrm{p}<0,05)$. Para la capacidad de oviposición, se hizo el análisis de regresión lineal para ambos parasitoides entre el número de días (variable independiente) y el número promedio de pupas parasitadas (variable dependiente) para determinar si existía algún tipo de relación lineal entre ambas. El diseño estadístico empleado fue un DBCR (Diseño en bloques completamente randomizado). Para $S$. endius fue 15 (días evaluados) x 10 (repeticiones) y para M. raptorellus fue 16 (días evaluados) x 10 (repeticiones). Los datos fueron transformados previamente al logaritmo $\mathrm{x}+1$. De los resultados del ANDEVA se hizo la prueba de Tukey $(\mathrm{p}<0,05)$. Los datos porcentuales de la determinación del período pupal preferido y número óptimo de pupas fueron transformados a la raíz cuadrada del arco seno antes de su análisis estadístico. En el primer caso se hizo el análisis de regresión para ambos parasitoides, entre el período de maduración de las pupas (variable independiente) y el número de pupas parasitadas (variable dependiente). El diseño estadístico empleado fue un DBCR. Después del ANDEVA, se efectuó la prueba de comparación múltiple siguiendo el criterio de Tukey. Para determinar el número óptimo de pupas, se realizó el análisis de regresión lineal para las dos especies, entre la densidad de pupas expuestas a la parasitación (variable independiente) y el número de pupas parasitadas (variable dependiente). Los resultados se expresaron en porcentaje de parasitismo con relación al número de pupas expuestas. Antes del análisis estadístico los datos porcentuales fueron transformados a la raíz cuadrada del arco seno, en un DCR. Establecida la significancia estadística del ANDEVA, se evaluó las diferencias entre los tratamientos, realizándose la prueba de Tukey. Para el cálculo de los estadísticos descriptivos e inferenciales se utilizó el paquete SPSS 12,0 en español para Windows. 


\section{RESULTADOS}

\section{Influencia de la temperatura en el ciclo biológico y longevidad de los parasitoides}

En el ciclo biológico. Utilizando diferentes temperaturas, se observó que la duración del ciclo biológico de ambas microavispas tiende a disminuir cuando se incrementan las temperaturas, lo que se evidenció con las ecuaciones de regresión lineal siguientes: $S$. endius $\left(\mathrm{Y}=53,05-0,97 \mathrm{X} ; \mathrm{F}=35,86 ; \mathrm{gl}=1\right.$ y $\left.2 ; \mathrm{R}^{2}=0,95\right) ; \mathrm{y} M$. raptorellus $\left(\mathrm{Y}=28,85-0,98 \mathrm{X} ; \mathrm{F}=61,84 ; \mathrm{gl}=1\right.$ y $\left.2 ; \mathrm{R}^{2}=0,97\right)$, donde $\mathrm{Y}=$ duración en días del ciclo biológico y $\mathrm{X}=$ temperatura de ensayo. La influencia de la temperatura en el ciclo biológico de estas especies puede observarse en el Cuadro 1. El ANDEVA resultó estadísticamente significativo al evaluar la duración del ciclo biológico de los dos parasitoides a las cuatro temperaturas experimentales $(\mathrm{F}=217,3 ; \mathrm{gl}=7$ y $72 ; \mathrm{P}=0,00)$. Al aplicarse la prueba de Tukey, se observó que la duración del ciclo biológico para $S$. endius y $M$. raptorellus fue diferente a cada temperatura ensayada. El análisis entre ambas especies a una misma temperatura, muestra en todos los casos, una mayor duración del ciclo biológico en $S$. endius, en contraste con $M$. raptorellus. A 25 y $30{ }^{\circ} \mathrm{C}$ el desarrollo fue significativamente más corto que a 15 y $20^{\circ} \mathrm{C}$.

Cuadro 1. Influencia de la temperatura en los ciclos biológicos de Spalangia endius y Muscidifurax raptorellus.

\begin{tabular}{ccccccc}
\hline $\begin{array}{c}\text { Temperatura } \\
\left({ }^{\circ} \mathrm{C}\right)\end{array}$ & \multicolumn{3}{c}{$\begin{array}{c}\text { Spalangia endius } \\
\mathrm{X} \pm \mathrm{DE}\end{array}$} & \multicolumn{3}{c}{ Muscidifurax raptorellus } \\
& \multicolumn{3}{c}{$\mathrm{X} \pm \mathrm{DE}$} \\
\hline 15 & 46,2 & \pm & $4,1 \mathrm{a}$ & 25,3 & \pm & $1,8 \mathrm{c}$ \\
20 & 31,6 & \pm & $2,5 \mathrm{~b}$ & 19,6 & \pm & $1,5 \mathrm{~d}$ \\
25 & 22,6 & \pm & $2,8 \mathrm{c}$ & 14,8 & \pm & $1,4 \mathrm{e}$ \\
28 & 17,9 & \pm & $1,6 \mathrm{~d}$ & 12,5 & \pm & $0,8 \mathrm{f}$ \\
\hline
\end{tabular}

$\mathrm{X}=$ Duración promedio del ciclo biológico en días.

$\mathrm{DE}=$ Desviación estándar.

En la longevidad. Se observó que la longevidad en ambas especies disminuyó conforme aumentó la temperatura utilizada (Cuadro 2). Esto es confirmado por las ecuaciones de regresión lineal siguientes que fueron estadísticamente significativas: S. endius $\left(\mathrm{Y}=77,67-0,99 \mathrm{X} ; \mathrm{F}=731,2 ; \mathrm{gl}=1\right.$ y $\left.2 ; \mathrm{R}^{2}=1,00\right) ;$ y M. raptorellus $\left(\mathrm{Y}=75,99-0,99 \mathrm{X} ; \mathrm{F}=272,00 ; \mathrm{gl}=12 ; \mathrm{R}^{2}=0,99\right)$, donde $\mathrm{Y}=$ longevidad (días) $\mathrm{y}$ 
$\mathrm{X}=$ temperatura de ensayo. El ANDEVA resultó estadísticamente significativo $(\mathrm{F}=168,8 ; \mathrm{gl}=7$ y $72 ; \mathrm{P}=0,00)$. Según la prueba de Tukey, la duración de la longevidad para ambas especies fue diferente con cada temperatura ensayada. Al analizar ambas especies a una misma temperatura, podemos observar que las longevidades son estadísticamente iguales.

Cuadro 2. Influencia de la temperatura en la longevidad de Spalangia endius y Muscidifurax raptorellus.

\begin{tabular}{ccc}
\hline $\begin{array}{c}\text { Temperatura } \\
\left({ }^{\circ} \mathrm{C}\right)\end{array}$ & $\begin{array}{c}\text { Spalangia endius } \\
\mathrm{X} \pm \mathrm{DE}\end{array}$ & $\begin{array}{c}\text { Muscidifurax raptorellus } \\
\mathrm{X} \pm \mathrm{DE}\end{array}$ \\
\hline 15 & $44,2 \pm 3,6 \mathrm{a}$ & $41,3 \pm 4,0 \mathrm{a}$ \\
20 & $31,4 \pm 3,4 \mathrm{~b}$ & $32,3 \pm 2,6 \mathrm{~b}$ \\
25 & $20,7 \pm 2,7 \mathrm{c}$ & $18,6 \pm 2,4 \mathrm{c}$ \\
28 & $14,6 \pm 2,2 \mathrm{~d}$ & $12,7 \pm 1,3 \mathrm{~d}$ \\
\hline
\end{tabular}

$\mathrm{X}=$ Duración promedio de la longevidad en días.

$\mathrm{DE}=$ Desviación estándar.

\section{Capacidad de oviposición}

Al comparar la capacidad de oviposición de ambas microavispas, se comprobó que hubo variación a través de los días de exposición de las pupas de M. domestica. S. endius tiende a un ligero incremento en la capacidad de oviposición que $M$. raptorellus y para ambas microavispas, esta capacidad tiende a disminuir con la longevidad de la hembra, esto se evidencia en las ecuaciones de regresión lineal que son estadísticamente significativas: Para $S$. endius $\left(\mathrm{Y}=19,12-0,94 \mathrm{X} ; \mathrm{F}=114,61 ; \mathrm{gl}=1\right.$ y $\left.14 ; \mathrm{R}^{2}=0,89\right)$; y $M$. raptorellus $\left(\mathrm{Y}=16,46-0,98 \mathrm{X} ; \mathrm{F}=270,3 ; \mathrm{gl}=1\right.$ y $\left.14 ; \mathrm{R}^{2}=0,95\right)$, donde $\mathrm{Y}=$ Número de pupas parasitadas y $\mathrm{X}=$ longevidad del parasitoide. El ANDEVA fue estadísticamente significativo $(\mathrm{F}=51,4 ; \mathrm{gl}=30$ y $279 ; \mathrm{P}=0,00)$. Según la prueba de Tukey, para $S$. endius a partir del $10{ }^{\mathrm{mo}}$ día existieron diferencias significativas en comparación con el tercer día que presentó la máxima capacidad de oviposición. Además en el día 10 y 15, se observó una mayor capacidad de oviposición de S. endius en comparación con M. raptorellus. Los demás días, las capacidades ovipositoras de ambas especies fueron similares. Para $M$. raptorellus al tercer día se notó la máxima capacidad de oviposición. Además, a partir del $7^{\text {mo }}$ día fue diferente del $3^{\text {er }}$ día de oviposición. Se observó 15 días de oviposición en $S$. endius, alcanzando un promedio de 175 pupas parasitadas, siendo mayor que $M$. raptorellus el cual parasitó sólo 140 pupas en 16 días. En el caso de S. endius, se pudo observar que en los días 9 y 13, su capacidad de oviposición aumenta numéricamente, aunque no estadísticamente significativa para luego seguir disminuyendo (Figuras 1 y 2). 


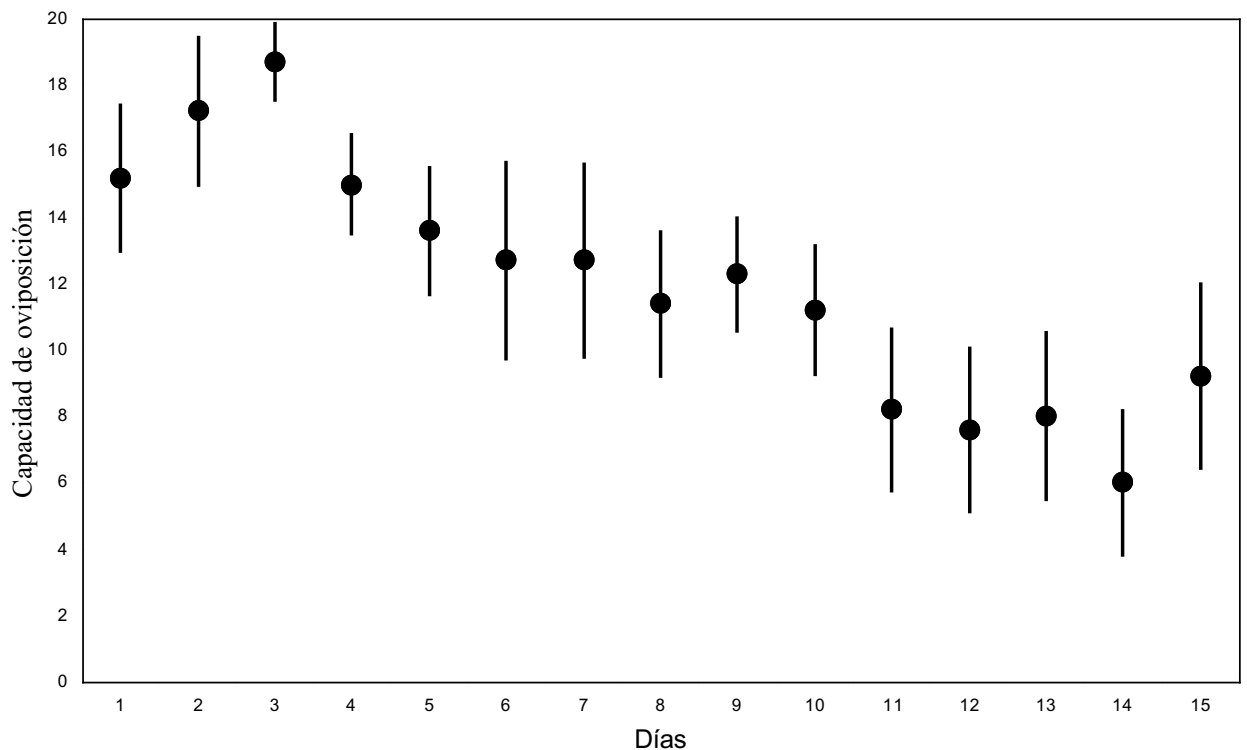

Figura 1. Comparación de la capacidad de oviposición de Spalangia endius a $25^{\circ} \mathrm{C}$.

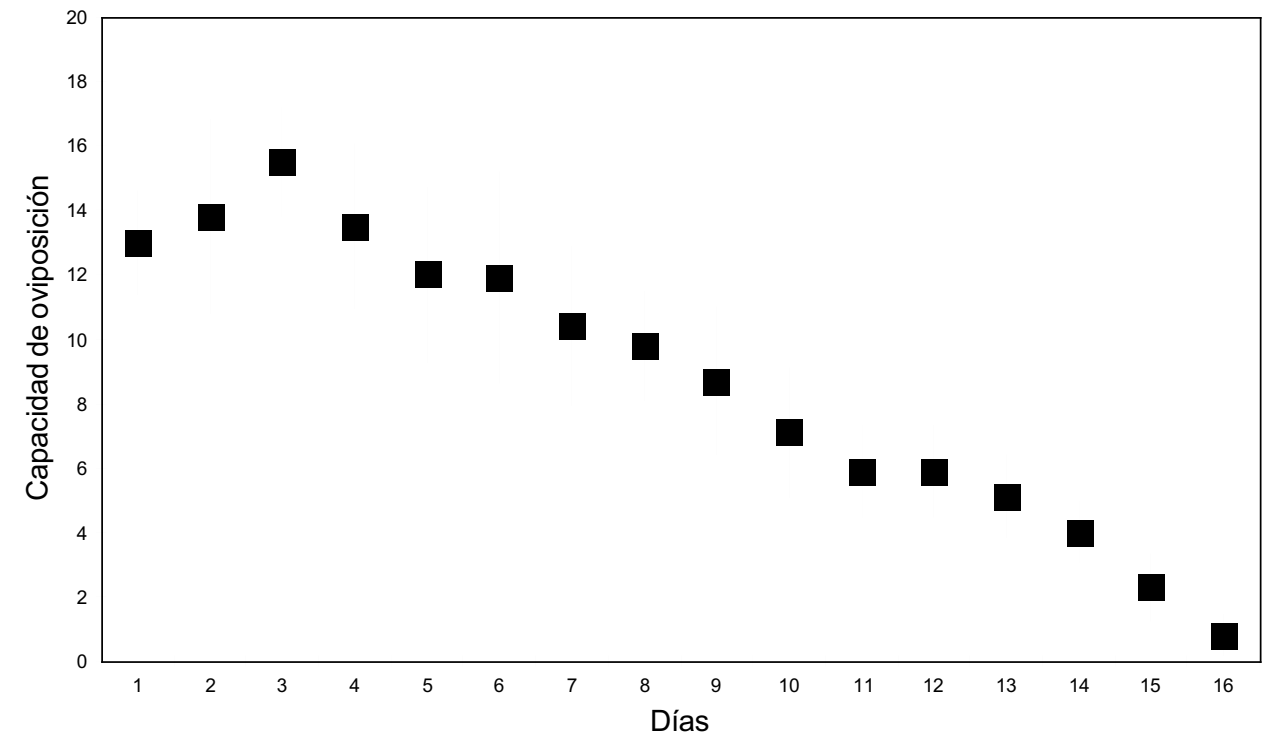

Figura 2. Comparación de la capacidad de oviposición de Muscidifurax raptorellus a $25{ }^{\circ} \mathrm{C}$. 


\section{Período pupal óptimo de $M$. domestica para ser parasitada}

Durante los cuatro estadíos pupales de las microavispas se observó que el parasitismo de las pupas varió según el estado de maduración de las mismas; sin embargo las ecuaciones de regresión lineal no fueron estadísticamente significativas: S. endius $\left(\mathrm{Y}=79,00-0,89 \mathrm{X} ; \mathrm{F}=7,81 ; \mathrm{gl}=1 \mathrm{y} 2 ; \mathrm{R}^{2}=0,8 ;\right)$ y $M$. raptorellus $\left(\mathrm{Y}=62,70-0,69 \mathrm{X} ; \mathrm{F}=1,78 ; \mathrm{gl}=1\right.$ y $\left.2 ; \mathrm{R}^{2}=0,47\right)$, donde $\mathrm{Y}=$ Período de maduración de las pupas (días) y $\mathrm{X}=$ Número de pupas parasitadas. Resultando estadísticamente significativas las diferencias en el porcentaje de pupas parasitadas en relación a la edad de la pupa según el $\operatorname{ANDEVA}(\mathrm{F}=18,6 ; \mathrm{gl}=7$ y $72 ; \mathrm{P}=0,00)$. (La prueba de Tukey dio para los parasitoides diferencias significativas en las pupas de 2 días en comparación con el resto. En las dos especies las menos parasitadas fueron las pupas de 4 días. Las pupas de 2 días fueron más parasitadas por ambas especies, obteniéndose un $66,4 \%$ de parasitismo efectivo por $S$. endius y un $60,2 \%$ por $M$. raptorellus por ello es que son la pupas más recomendables para ser parasitadas. Se observó un menor parasitismo en las pupas de 4 días en ambas especies, $32 \%$ en $S$. endius y $18,8 \%$ en M. raptorellus (Cuadro 3 ).

Cuadro 3. Comparación del período pupal óptimo de Spalangia endius y Muscidifurax raptorellus.

\begin{tabular}{ccc}
\hline \multirow{2}{*}{ Días } & Spalangia endius & Muscidifurax raptorellus \\
\cline { 2 - 3 } & $\mathrm{X} \pm \mathrm{DE}$ & $\mathrm{X} \pm \mathrm{DE}$ \\
\hline 1 & $61,8 \pm 16,6 \mathrm{ab}$ & $42,0 \pm 8,3 \mathrm{~cd}$ \\
2 & $66,4 \pm 13,3 \mathrm{a}$ & $60,2 \pm 13,6 \mathrm{ab}$ \\
3 & $49,6 \pm 11,6 \mathrm{bc}$ & $39,8 \pm 10,3 \mathrm{~cd}$ \\
4 & $32,0 \pm 11,5 \mathrm{de}$ & $18,8 \pm 7,5 \mathrm{e}$ \\
\hline
\end{tabular}

$\mathrm{X}=$ Promedio de pupas parasitadas.

$\mathrm{DE}=$ Desviación estándar.

\section{Comparación del número óptimo de pupas de $M$. domestica para ser parasitadas con ambas especies}

Se observó que el parasitismo en ambas microavispas, estuvo influenciado significativamente por el número de pupas expuestas al parasitismo entre los límites de 10 y 50 pupas de mosca/hembra del parasitoide. Esto concuerda con las ecuaciones de regresión lineal que son estadísticamente significativas, con una tendencia a disminuir el porcentaje de pupas parasitadas con el incremento de pupas expuestas a ambos parasitoides: $S$. endius $(\mathrm{Y}=94,65-0,76 \mathrm{X} ; \mathrm{F}=9,29 ; \mathrm{gl}=1$ y 7 ; 
$\left.\mathrm{R}^{2}=0,57\right)$ y M. raptorellus $\left(\mathrm{Y}=97,85-0,95 \mathrm{X} ; \mathrm{F}=68,3 ; \mathrm{gl}=1\right.$ y $\left.8 ; \mathrm{R}^{2}=0,90\right)$ donde $\mathrm{Y}=$ Número de pupas parasitadas y $\mathrm{X}=$ densidad de pupas expuestas. El ANDEVA resultó estadísticamente significativo $(\mathrm{F}=24,4 ; \mathrm{gl}=17$ y $162 ; \mathrm{P}=0,00)$. El número óptimo de pupas para exponer a ambos parasitoides fue 10 (relación 1:10). La Prueba de comparación múltiple de Tukey, nos muestra que las relaciones 1:5 y 1:10 fueron diferentes para ambos parasitoides. El análisis entre ambas especies a una misma densidad de pupas, muestra que en todas las relaciones pareja de parasitoides: pupas de mosca, excepto en la última (relación 1: 50) no existieron diferencias significativas (Cuadro 4).

Cuadro 4. Comparación del número óptimo de pupas susceptibles a Spalangia endius y Muscidifurax raptorellus y su relación con el porcentaje de pupas parasitadas.

\begin{tabular}{ccc}
\hline $\begin{array}{c}\text { Relación: } \\
\text { parasitoide: } \\
\text { pupa }\end{array}$ & Spalangia endius & Muscidifurax raptorellus \\
\cline { 2 - 3 } & \multicolumn{1}{c}{$\mathrm{X} \pm \mathrm{DE}$} & $\mathrm{X} \pm \mathrm{DE}$ \\
\hline $1: 5$ & $100,0 \pm 0,0 \mathrm{a}$ & $98,0 \pm 6,3 \mathrm{a}$ \\
$1: 10$ & $96,0 \pm 6,9 \mathrm{a}$ & $91,0 \pm 11,0 \mathrm{a}$ \\
$1: 15$ & $71,9 \pm 14,3 \mathrm{bc}$ & $69,3 \pm 12,6 \mathrm{bc}$ \\
$1: 20$ & $84,0 \pm 6,1 \mathrm{ab}$ & $73,0 \pm 11,3 \mathrm{bc}$ \\
$1: 25$ & $65,2 \pm 13,6 \mathrm{~cd}$ & $60,0 \pm 9,8 \mathrm{cde}$ \\
$1: 30$ & $61,2 \pm 10,4 \mathrm{cde}$ & $56,0 \pm 9,3 \mathrm{cde}$ \\
$1: 35$ & $58,3 \pm 12,6 \mathrm{cde}$ & $46,9 \pm 13,8 \mathrm{e}$ \\
$1: 40$ & $62,2 \pm 15,7 \mathrm{cde}$ & $48,5 \pm 12,1 \mathrm{de}$ \\
$1: 50$ & $70,6 \pm 13,5 \mathrm{bc}$ & $46,4 \pm 8,6 \mathrm{e}$ \\
\hline
\end{tabular}

$\mathrm{X}=$ Porcentaje de pupas parasitadas.

$\mathrm{DE}=$ Desviación estándar.

\section{DISCUSIÓN}

\section{Influencia de la temperatura en la crianza de los parasitoides}

Ciclo biológico. Los valores hallados por la mayoría de autores, coinciden con los obtenidos en este trabajo, las pequeñas diferencias pueden ser explicadas debido a ciertas variaciones en las condiciones de la crianza de los parasitoides, como humedad y temperatura. Moreno (1980) al estudiar la biología comparada de ambas microavispas encontró que a $26^{\circ} \mathrm{C}$ las hembras de $S$. endius tuvieron un ciclo de 25 días y los machos, 23 días; mientras que las hembras de $M$. raptorellus tuvieron un ciclo biológico de 18 
días y los machos, de 17 días. En nuestro estudio, la duración del ciclo biológico fue de 22,6 días y para $M$. raptorellus fue 14,8 días. Como puede observarse para el caso de $S$. endius obtuvimos valores inferiores a los Moreno (op cit.). Mamani (1984), a $25{ }^{\circ} \mathrm{C}$ reporta la duración del ciclo biológico de $S$. endius en 21,5 días. A mayor temperatura el ciclo se hizo más corto y también el rango, para ambos sexos, datos que coinciden con lo reportado por Moreno (1980) y Mamani (1984). A $15{ }^{\circ} \mathrm{C}$ se observa mejor la emergencia de los parasitoides adultos y es recomendable esta temperatura con fines de conservación de pupas de moscas parasitadas, en un sistema de producción masiva. También se han reportado algunos datos sobre estudios de los ciclos biológicos de otras especies de Spalangia y Muscidifurax.; así, Kogan \& Legner (1970) reportan entre 22 y 24 días el ciclo biológico de M. raptor y para M. raptorellus una duración de entre 17 a 21 días a $26 \pm 1{ }^{\circ} \mathrm{C}$ y $75 \pm 5 \mathrm{HR}$. Al estudiar el ciclo de vida y hábitos de Spalangia cameroni Perkins, Ortiz y Torres (1983) observaron que el ciclo biológico de esta especie duraba 15 días. Morgan et al. (1991) encontraron para Spalangia gemina Boucek que duraba 22 días a 25,5 ${ }^{\circ} \mathrm{C}$. Mann et al. (1990b) encontraron para Muscidifurax raptor Kogan \& Legner, M. zaraptor Kogan \& Legner, S. cameroni y $S$. endius, una duración del ciclo biológico de 20,7 días, 22,8 días, 35,8 días y 31,5 a $25^{\circ} \mathrm{C}$.

Longevidad. La longevidad de los parasitoides, está muy asociada con el tipo de alimentación. Se observó que las hembras se alimentan además de la miel que se les dio como dieta diaria, de los exudados de la pupa hospedera luego de parasitarla, sin afectar el desarrollo del parasitoide hijo. Los valores de longevidad obtenidos por Legner et al. (1967) y Mamani (1984) para S. endius coinciden con el rango y aproximadamente con los valores promedios encontrados en este ensayo, en el que a $25{ }^{\circ} \mathrm{C}$, obtuvimos longevidades de 20,7 días en $S$. endius y 18,6 días en $M$. raptorellus. Las hembras de $S$. endius tuvieron una longevidad de 22 días y los machos 19,4 días; las hembras de M. raptorellus tuvieron una longevidad de 24,6 días y los machos 17,6 días. Legner et al. (1967), señala que la longevidad promedio de la hembra de $S$. endius es de 22 días al ser criadas en un ambiente entre 23 a 27 ${ }^{\circ} \mathrm{C}$ (Mamani 1984). Mamani (1984), señala que la longevidad de $S$. endius, a $25{ }^{\circ} \mathrm{C}$ es de 26,2 días para las hembras y de 19,4 días para los machos. A diferencia de estos valores, Moreno (1980), encontró a $26{ }^{\circ} \mathrm{C}$, una longevidad promedio de 8 días para S. endius y 8,5 días para M. raptorellus Legner \& Gerling (1967) al trabajar con $S$. cameroni encontraron que a $26^{\circ} \mathrm{C}$, la longevidad de este parasitoide varía entre 24 y 27 días.

\section{Capacidad de oviposición de $S$. endius y M. raptorellus}

Esta capacidad en estos parasitoides no ha sido muy estudiada. En el Perú sólo se tienen trabajos de Mamani (1984) y Polanco (1996) cuyos valores coinciden con el nuestro. Dentro de los primeros seis días $S$. endius aportó el $52,80 \%$ del total de oviposiciones, en $M$. raptorellus fue del 57,06\%. Mamani (1984) obtuvo un 
promedio de 196 parasitoides de $S$. endius a $19^{\circ} \mathrm{C}$ y Polanco (1996) un promedio de 163,8 a la misma temperatura. En ambos parasitoides, el tercer día de su emergencia las hembras parasitaron un mayor número de pupas, coincidiendo también en esto, con los trabajos de Mamani (1984) y Polanco (1996). La capacidad de oviposición depende en gran medida de la alimentación del parasitoide. La hembra al alimentarse del huésped logra una mayor fecundidad. La dieta que se le dio (miel de abeja) contribuyó significativamente para ello, además de las condiciones de temperatura y oscuridad. Como puede observarse, el total de pupas parasitadas en $S$. endius es mayor que en $M$. raptorellus, esto puede deberse a las características biológicas de $S$. endius de poder parasitar hasta a una profundidad de $20 \mathrm{~cm}$ y de "marcar" pupas ya parasitadas, capacidad de la que carece la otra especie. Mann et al. (1990b) encontraron a $25^{\circ} \mathrm{C}$ y a 1 día de exposición para $M$. raptor, M. zaraptor, $S$. cameroni y $S$. endius una capacidad de oviposición de $11 ; 8,9 ; 11$ y 3,4 pupas parasitadas, respectivamente.

\section{Período pupal óptimo de $M$. domestica para ser parasitada}

La susceptibilidad mayor al parasitismo ocurre cuando las pupas aún no se esclerotizan, asimismo cuando la intensidad de la luz es tenue. El máximo parasitismo de $S$. endius (25,3\%) se presentó en pupas de 1 día de edad y el mínimo, en pupas de 3 días (Moreno 1980). En el presente estudio, la mayor susceptibilidad (66,40\% en $S$. endius y $60,20 \%$ en $M$. raptorellus), se observó en pupas de 2 días. Ortíz \& Torres (1983) quienes al trabajar en el laboratorio con S. cameroni, obtuvieron mayor susceptibilidad (77\%) en pupas de 2 días; el $47 \%$, $28 \%$ y $23 \%$ en pupas de 1, 3 y 4 días respectivamente, en condiciones de temperatura de $26 \pm 1$ ${ }^{\circ} \mathrm{C}$ y $75 \pm 5 \%$ de humedad relativa. Como puede observarse, a medida que las pupas van madurando, se hacen menos "atractivas" a los parasitoides, en consecuencia, son menos parasitadas. Nuestros resultados son opuestos a lo encontrado Kaufman et al. (2001) para Nasonia vitripennis Walter y M. raptorellus, que indican el más bajo parasitismo en pupas de 2 días de edad.

\section{Número óptimo de pupas de $M$. domestica para ser expuestas a los parasitoides}

Tanto en $S$. endius como en $M$. raptorellus, las menores poblaciones $(5,10,15$ y 20) fueron relativamente más parasitadas que las poblaciones más altas del hospedero (25-50); existiendo los valores más altos en las primeras cuatro relaciones $(1: 5 ; 1: 10 ; 1: 15$ y $1: 20)$. Esta relación inversa del parasitismo con respecto al número de pupas expuestas fue hallada antes por Ables \& Shepard (1974) quienes al estudiar a $S$. endius, reportaron una población de 30 pupas, un $98 \%$ de parasitismo y con 80 pupas, $67 \%$ de parasitismo entre 25 y $29^{\circ} \mathrm{C}$. Ortiz \& Torres (1983) consideran una población de 10 pupas como la más óptima para la parasitación por $S$. cameroni a 26 $\pm 1{ }^{\circ} \mathrm{C}$ y $75 \pm 5 \%$ de humedad relativa. Al igual que Mann et al. (1990a) que 
encontró la mejor parasitación por M. raptor y S. cameroni en la relación 1:10. En otros estudios biológicos, con especies del género Spalangia, Moreno (1980) empleó 10 pupas de mosca por parasitoide hembra al día y Legner et al. (1967), 20 pupas por parasitoide hembra al día (Mamani 1984). Morgan et al. (1991) encontraron para $S$. gemina en la relación 1:10, un parasitismo de 81-87\% en $M$. domestica.

En el Perú, un programa eficiente de control de $M$. domestica requiere la liberación de las avispas $S$. endius y $M$. raptorellus, dependiendo del tipo de explotación pecuaria y de las densidades poblacionales de la mosca (Meyer et al. 1991). También se debe implementar otras acciones complementarias como el manejo de excretas, de aguas residuales y de olores, así como el control de moscas adultas con trampas con atrayentes alimenticios. Ambas microavispas al tener un ciclo de vida corto, no son fáciles de establecerse en el medio ambiente, razón por la cual se deben liberar periódicamente (Rutz et al. 1989). En este programa es posible alcanzar hasta un $90 \%$ de control de moscas en explotaciones pecuarias después de 90 a 120 días de iniciado el mismo.

\section{LITERATURA CITADA}

Ables, J. \& M. Shepard. 1974. Responses and competition of the parasitoids Spalangia endius and Muscidifurax raptor (Hymenoptera: Pteromalidae) at different densities of house fly pupas. Can. Entomol. 106:825-830.

Atias, A. 1995. Parasitología Clínica. Tercera Ed. Santiago de Chile. 618 pp.

Díaz, L., J. Luque \& H. Calvache. 1996. Estudios básicos para un manejo integrado de la mosca de los establos, Stomoxys calcitrans L. (Diptera: Muscidae). Rev. Col. Entomol. 22:77-85.

Geden, C. 1998. Host location by five species of parasitoids (Hymenoptera: Pteromalidae, Chalcididae) of house flies, Musca domestica L. (Díptera: Muscidae), in poultry manure as a function of moisture levels and host density. United States Department of Agriculture $326 \mathrm{pp}$.

Kaufman, P.E, S.J. Long \& Rutz, D.A. 2001. Impact of exposure length and pupal source on Muscidifurax raptorellus and Nasonia vitripennis (Hymenoptera: Pteromalidae) parasitism in a New York poultry facility. J. Econ. Entomol. 94: 998-1003.

Keiding, J. 1987. La Mosca Doméstica: Biología y Control. Documento de la Organización Mundial de la Salud. OMS-937. 69 pp.

Kogan, M. \& E. Legner. 1970. A biosystematic revision of the genus Muscidifurax (Hymenóptera: Pteromalidae) with descriptions of four new species. Can. Entomol. 102:1268-1290.

Legner, E. 1967. Behavior changes the reproduction of Spalangia cameroni, S. endius, Muscidifurax raptor, and Nasonia vitripennis (Hymenoptera: Pteromalidae) at increasing fly host densities. Ann. Entomol. Soc. Am. 60:819-826.

Legner, E., E. Bay \& E. White. 1967. Activity of parasites from Diptera: M. domestica, $S$. calcitrans, Fannia canicularis, and F. femoralis at sites in the Western Hemisphere. Ann. Entomol. Soc. Am. 60:462-468. 
Legner, E. \& D. Gerling. 1967. Host-feeding and oviposition on Musca domestica by Spalangia cameroni, Nasonia vitripennis, and Muscidifurax raptor (Hymenóptera: Pteromalidae) influences their longevity and fecundity. Ann. Entomol. Soc. Am. 60:678-691.

Mamani, J. 1984. Efectos de la temperatura en la capacidad reproductiva y en el ciclo de desarrollo de Spalangia endius Walker (Hymenoptera: Pteromalidae), parásito pupal de la mosca doméstica. Tesis de Maestría. Universidad Nacional Agraria la Molina. Lima. Perú. $111 \mathrm{pp}$.

Mann, J.A., R.E. Stinner \& R.C. Axtell. 1990a. Parasitism of house fly (Musca domestica) pupae by four species of Pteromalidae (Hymenoptera): effects of host-parasitoid densities and host distribution. Med. Vet. Entomol. 4:235-243.

Mann, J.A., R.C. Axtell \& R.E. Stinner. 1990b. Temperature-dependent development and parasitism rates of four species of Pteromalidae (Hymenoptera) parasitoids of house fly (Musca domestica) pupae. Med. Vet. Entomol. 4:245-253.

Manrique, P. \& H. Delfín. 1997. Importancia de las moscas como vectores potenciales de enfermedades diarreicas en humanos. Rev. Biomédica 8:163-170.

Meyer, J. A., T. A. Schutz, C. Collar, \& B. A. Mullens. 1991. Relative abundance of stable and house fly pupal parasites on confinement dairies in California. Environ. Entomol. 20: 915-921.

Moreno, E. 1980. Biología comparada de Muscidifurax sp. y Spalangia endius (Hymenoptera: Pteromalidae) ectoparásitos pupales de la mosca doméstica (Musca domestica). Tesis de Pre grado. Universidad Particular Ricardo Palma. Lima. Perú. 98 p.

Morgan, P.B., E. Berti-Filho \& V.A. Costa. 1991. Life history of Spalangia gemina Boucek (Hymenoptera: Pteromalidae), a fast-breeding microhymenopteran pupal parasitoid of muscoid flies. Med. Vet. Entomol. 5:277-281.

Mullens, B. A., J. A. Meyer \& J. D. Mandeville. 1986. Seasonal and diel activity of filth fly (Musca domestica) in caged-layer poultry manure in sourthern California. Environ. Entomol. 15: 56-60.

Ortíz, R. \& J. Torres. 1983. Ciclo de vida y hábitos de Spalangia cameroni Perkins (Hymenoptera - Pteromalidae) en condiciones de Laboratorio. Tesis de Pregrado. Pro - Perkins Ltda. Palmira. 57 p.

Patterson, R. S. \& D. A. Rutz. 1986. Biological Control of muscoid flies. Misc. Publ. Entomol. Soc. Am. 61:1-174.

Polanco, M. 1996. Crianza de Spalangia endius Walker (Hymenoptera: Pteromalidae) parásito pupal de la mosca doméstica en condiciones de laboratorio. Tesis de Pre grado. Universidad Nacional San Agustín. Arequipa. Perú. 36 pp.

Rutz, D. A. \& G. A. Scoles. 1989. Occurrence and seasonal abundance of parasites attacking muscoid flies (Diptera: Muscidae) in caged-layer poultry facilities in New York. Environ. Entomol. 18: 51-55.

Vergara, R. \& J. Jiménez. 1995. Manejo integrado de moscas comunes (MIMD) en explotaciones pecuarias y salud pública con énfasis en control biológico. Pp. 347- 359. In: Gomero L, Lizárraga A. (Eds.). Aportes del Control Biológico en la Agricultura Sostenible. Lima, Perú.

Weinzierl, R.A. \& C.J. Jones. 1998 Releases of Spalangia nigroaenea and Muscidifurax zaraptor (Hymenoptera: Pteromalidae) increase rates of parasitism and total mortality of 
Inciso \& Iannacone: Parasitoides en Musca domestica

stable fly and house fly (Diptera: Muscidae) pupae in Illinois cattle feedlots. J. Econ. Entomol. 91:1114-1121.

Zamora, E. 1996. Técnica de Producción masiva de Spalangia endius Walker. pp. 125 - 129. II Curso de Actualización en Control Biológico. Centro Nacional de Referencia de Control Biológico. México.

Recibido: 18 de febrero de 2007

Aceptado: 6 de febrero de 2008 